\title{
Age Estimation for Dental Patients Using Orthopantomographs
}

\author{
Bekir Karaarslan ${ }^{a}$ \\ Emine Sirin Karaarslan ${ }^{b}$ \\ Abdul Semih Ozsevik ${ }^{c}$ \\ Ertan Ertas $^{c}$
}

\section{ABSTRACT}

Objectives: The aim of this study was to conduct age estimates for dental patients using orthopantomographs (OPGs). The OPGs were selected by an independent author with respect to criteria and evaluated by two independent dentists. The results were compared to chronologic ages. The reliability of the estimates, concurrently made by the two independent dentists using OPGs, was also evaluated.

Methods: In this retrospective study, the OPGs of 238 Turkish individuals of known chronological age, ranging from 1 to 60 years, were measured. Patients were then classified. Radiographs were evaluated by two independent dentists and age estimation was achieved according to the decades.

Results: The truest age estimates made by the dentists were in the 1-10 years age range (89.6\%), the most inaccurate age estimates were in the $41-50$ years age range $(41.7 \%)$. Results indicate that the accuracy of age estimation diminishes with age.

Conclusions: Despite the variations related to the practitioners, in this study, there were no significant differences in age estimations between the two participant practitioners. Age estimation through evaluating OPGs was the most accurate in the first decade and the least in fourth decade. It can be concluded that OPGs are not adequate for accurate age estimation. (Eur J Dent 2010;4:389394)

Key words: Age estimation; Forensic dentistry; Orthopantomographs.

a Institution of Forensic Medicine of the Ministry of Justice, Gaziantep Branch of the Forensic Medicine, Specialist of Forensic Medicine Gaziantep, Turkey.

b Department of Restorative Dentistry, Faculty of Dentistry, Gaziantep University, Gaziantep, Turkey. Department of Restorative Dentistry, Faculty of Dentistry, Ondokuz Mayis University, Samsun, Turkey.

- Corresponding author: Emine Sirin Karaarslan Department of Restorative Dentistry, Faculty of Dentistry, Gaziantep University, 27310, Gaziantep, Turkey. Phone: +90 342 3606060/76614 Fax: +90 3423610610 E-mail: karaarslan.emineagmail.com

\section{INTRODUCTION}

The forensic age estimation of unidentified skeletons and corpses for the purpose of identification has been a conventional feature of forensic science. Determining the identity of a decedent is of considerable significance from the ethical, legal, and criminal perspectives; not only is it the prerequisite for officially declaring an individual dead but it is also the basis for dealing with mass disasters, crimes, and war crimes. ${ }^{1}$ 
Compared to bone mineralization, tooth mineralization stages are much less affected by variation in endocrine and nutritional status, and developing teeth therefore provide a more certain indication of chronological age. ${ }^{2}$ Tooth formation is used often to assess maturity and predict age. Within clinical dentistry, this information aids in diagnosis and treatment planning. In forensic odontology and archaeology, age estimation methods can aid the identification of age at death of a deceased child and also give important information with regard to past populations. Age estimation is also proving valuable when birth data is lacking or doubted in the management of immigration to help determine physiological age. ${ }^{3}$

The scientific basis of age estimation is the genetic control of ontogenesis, which delimits the temporal variation of developmental stages. ${ }^{4} \mathrm{Ac}$ cording to the suggestions produced by the Study Group on Forensic Age Diagnostics, ${ }^{1}$ a forensic age estimate of a living person for the purpose of criminal prosecution should consist of: a physical examination that also records anthropometric data, any age-relevant developmental disorders and signs of sexual maturation; an X-ray examination of the left hand; and a dental examination that records dentition status and evaluates an orthopantomograph.

Several methods for the determination of dental development from radiographs have been described. ${ }^{5}$ In children and adolescents, age estimations are based on the developmental stage of the deciduous and permanent dentition. ${ }^{6}$ Most of these are based on a comparison of the radiographic development of teeth with standard diagrams collected from a large number of persons, usually in a well-defined geographic region. ${ }^{7}$ With advancing age, secondary dentine is deposited along the wall of the dental pulp chamber, leading to a reduction in the size of the pulp cavity. ${ }^{8}$ Tooth wear has been widely used as a tool of age estimation. ${ }^{9}$ The mechanisms by which teeth wear include attrition, abrasion, and erosion. Tooth attrition is defined as gradual and regular loss of tooth substance as a result of chewing activity. ${ }^{10}$ Age-related changes can be evaluated from dental radiographs. ${ }^{11}$

This study aimed to determine possibility of age estimation with the orthopantomographs (OPGs); the difference between the evaluations of
OPGs by dentists and the decade provide the most accurate age estimation.

\section{MATERIALS AND METHODS}

In this retrospective study, panoramic radiographs of 238 Turkish people of known chronological age, with ages ranging from 1 to 60 years, were enrolled. The OPGs taken at the 19 Mayis University, Faculty of Dentistry, Department of Oral Diagnosis were selected by an independent author with respect to convenient criteria. These OPGs were evaluated by two independent dentists at the same time interval. The age estimation for each case was done according to decade. Then the estimation results were compared to the chronological age of individuals by third independent dentist. Two correct estimations were recorded as "true-true," false estimations recorded as "falsefalse," and one correct and one false result were recorded as "correct-false."

The OPGs were selected according to the criteria $^{12}$ listed below:

- Both mandibular branches had to be of equal width

- The "Spee" curve had to appear concave

- The depth of field should be given

Patients were classified:

Group 1: 1-10 years

Group 2: 11-20 years

Group 3: $21-30$ years

Group 4: 31-40 years

Group 5: 41-50 years

Group 6: $51-60$ years

The evaluation criteria for the OPGs for both of dentists were compiled from the literature. ${ }^{13,14}$

- Presence of primary teeth in mouth

- Mixed dentition period

- Presence of third molar teeth in mouth

- Apexogenesis and maturation stage of third molar teeth

- Enamel attrition level of teeth

- Width of root canal and pulp cavity

- Level of alveolar bone resorbtions were considered during age estimation

The number and ratio of true and false predictions of two dentists were evaluated, and similarities and/or differences among them were calculated using a chi-square test. 


\section{RESULTS}

Among the 238 OPGs evaluated for age estimation, the first dentist predicted 174 of the cases $(73.1 \%)$ orthopantomographs correctly and 64 of the cases (26.9\%) incorrectly. The second dentist predicted 171 of the cases $(71.8 \%)$ correctly and 67 of the cases (28.2\%) incorrectly. The two clinicians evaluated 238 radiographs and predicted 149 of the cases (62.9\%) correctly and 42 of the cases $(17.6 \%)$ incorrectly. While evaluating the OPGs together, 47 of the cases (19.8\%) were evaluated correctly by one dentist and incorrectly by the other. The percentage of true and false age estimations of both dentists is shown in Figure 1 and Table 1. There was no significant difference between the dentists in terms of age estimation $(P>.05)$, and the accuracy of age estimation in the age groups was found to be high $(\lambda=0.73)$.
The greatest accuracy in the age estimations of the dentists occurred in the range of $1-10$ years of age $(89.6 \%)$, and the greatest percentage of incorrect age estimations occurred in the range of 41-50 years of age $(41.7 \%)$. The correct age estimation percentage diminished in the older age ranges. The evaluation criteria were given to the OPGs Figures 2 and 3.

\section{DISCUSSION}

Age estimation plays an important role in forensic medicine, clinical dentistry, pediatric endocrinology, and archaelogy. ${ }^{15}$ Age estimation is of wider importance in forensic medicine, not only for the purpose of identifying deceased victims but also in connection with crimes and accidents. In addition, chronological age is important in most societies for school attendance,

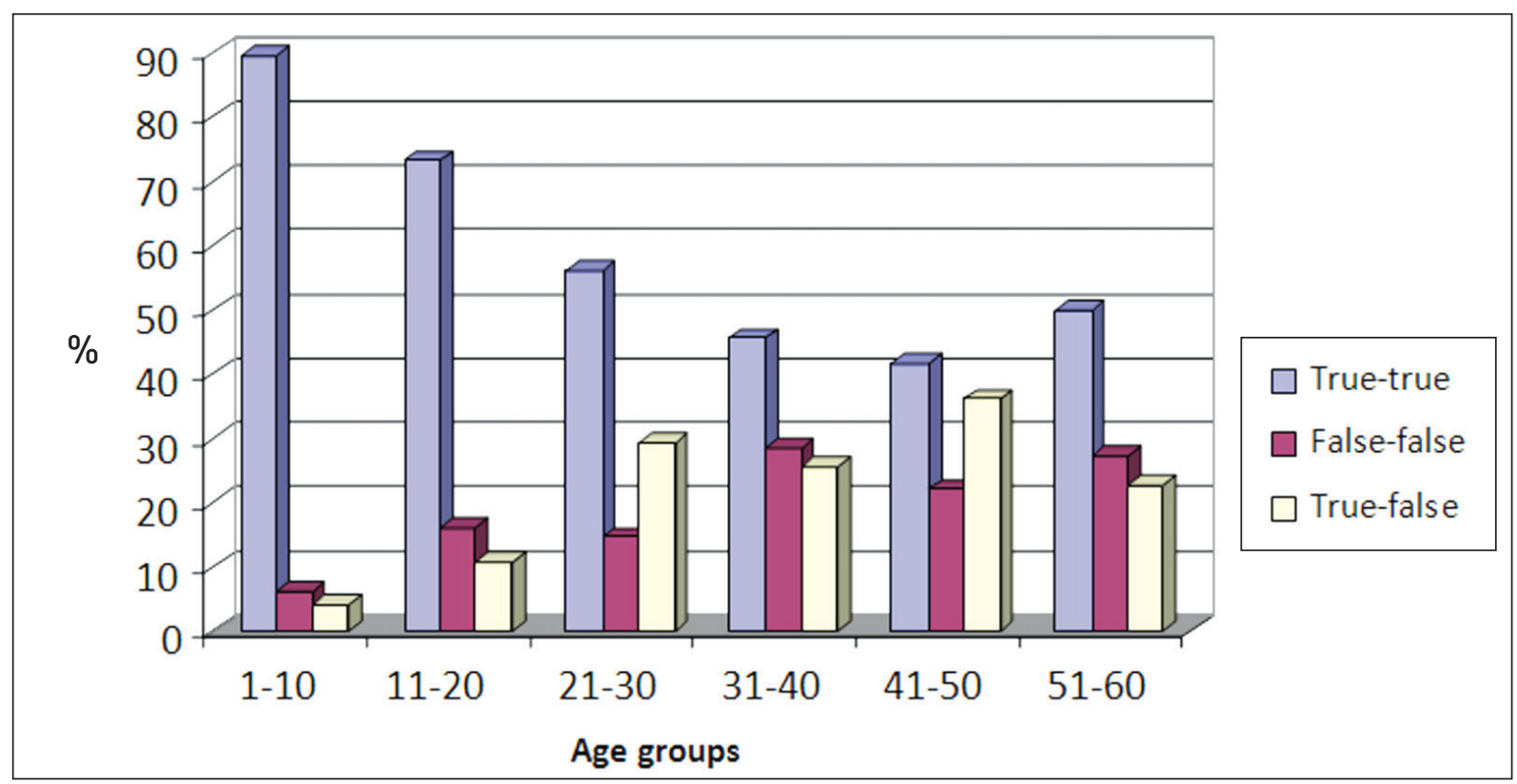

Figure 1. Distribution of true and false estimation percentage in accordance with age groups.

Table 1. Predictions of dentists for age estimation according to age groups.

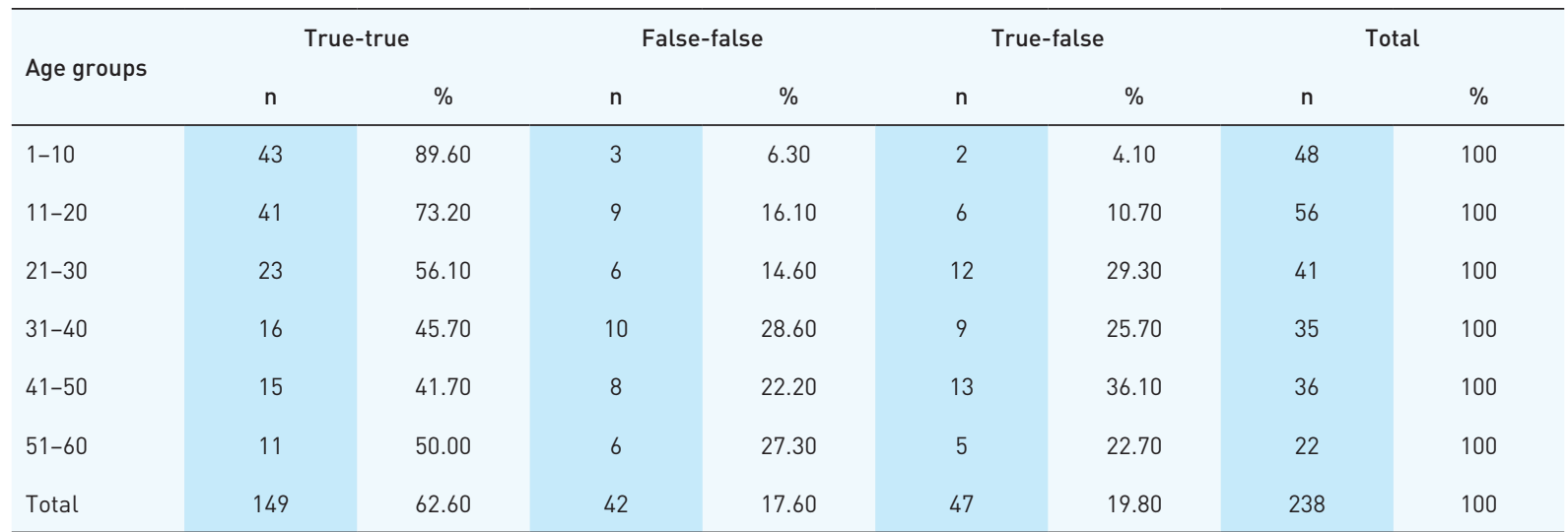

$\mathrm{n}$ : number of orthopantomographs. 
employment, social benefits, and marriage. ${ }^{2}$ In adulthood, teeth undergo time-related changes representing biological aging, and many studies have shown that several features of aging can be used for age determination. ${ }^{12}$ Gustafson ${ }^{6}$ developed the first dental method of age estimation in adults based on six criteria: attrition, secondary dentine inthe pulp, cementum annulations, root resorption, periodontal recession, and root translucency.

The methods in this study can be applied to living persons. Furthermore, OPGs also provide information regarding individuals' identity ${ }^{16}$ and other age-related features such as enamel attrition, secondary dentine in the pulp, root resorption, cementum annulations, and periodontal recession. ${ }^{13}$ In our study, the age groups were determined and age estimation was made in accordance with these groups.

In the present study, considering the necessity of identification and accurate age estimation of cases in daily practice of forensic medicine lage estimation for marriage, penal trials, and legal actions), and in the identification of disaster victims, a subjective age estimation method was implemented by evaluating OPGs.

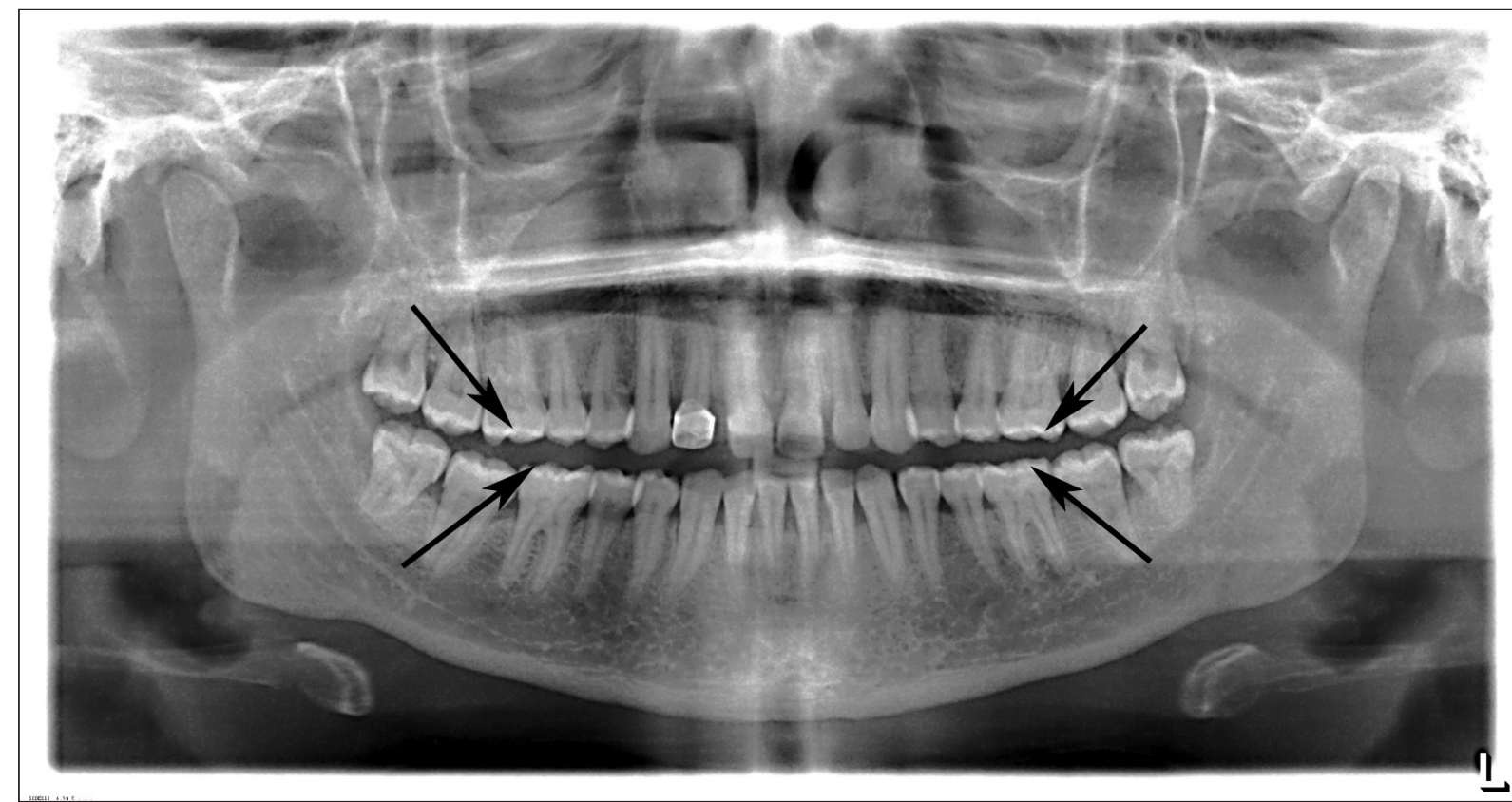

Figure 2. Attrition in enamel (Criteria for evaluating orthopantomographs).

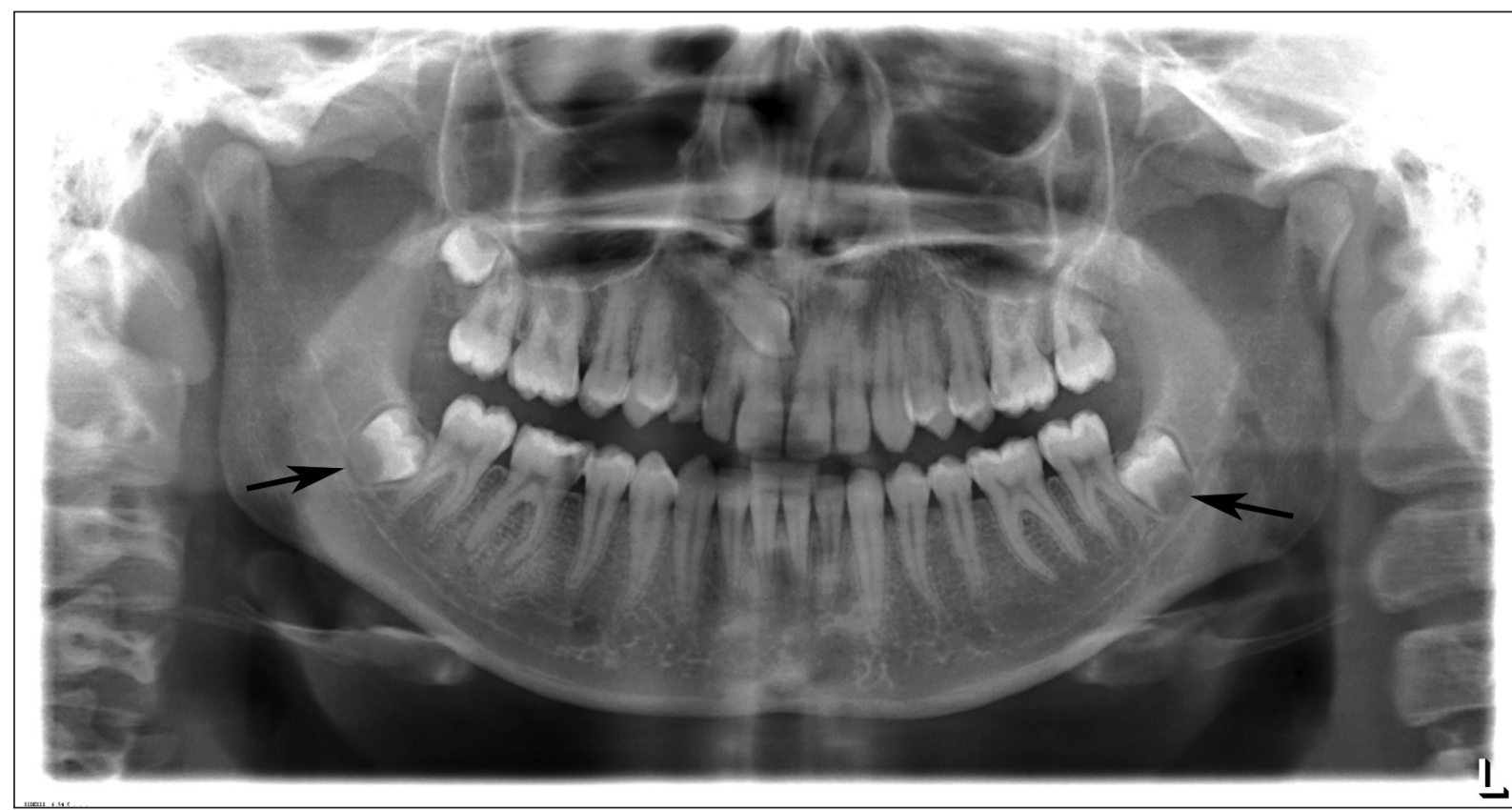

Figure 3. Apexogenesis and maturation stage of third molar teeth. 
Tooth improvement is a continuous process, but determining the end point of tooth development is very difficult. Thus, the calculation of a mean age for each phase is difficult; further research is needed to determine the apex closure stage of teeth. ${ }^{17}$ Measurement using dental radiographs may be useful as a non-invasive technique for estimating the age of adults, both living and dead, in archaeological studies and in forensic work, but the method should be tested on an independent sample. ${ }^{18}$

A scoring system for age estimation should be developed to ensure good reliability. To minimize the influence of intra-and inter examiner variations, well-defined criteria and careful calibration among examiners are esential. ${ }^{19}$ Even though the coronal pulp might be affected more by external factors than the root pulp, the scoring systems gave OPGs a strong correlation between age and the amount of secondary dentin for the coronal and for measurements in the root area. Age estimations based on measurements of the amount of secondary dentin present seem to be relatively reliable. ${ }^{20}$ Methods of age estimation in adults are concerned, and in view of the relative accuracy of the age estimations performed, one should keep in mind that the standard deviations of such age estimations are, in general, about 10 to 12 years. ${ }^{2}$

In this study, a scoring system was not used. Age estimation was made using criteria such as the presence of primary teeth in the mouth, mixed dentition period, presence of third molar teeth in the mouth, apexogenesis and maturation stage of third molar teeth, enamel attrition level of teeth, width of root canal and pulp cavity, and level of alveolar bone resorbtions. However, no measurements were made. Both independent dentists examined the OPGs at the same time. Estimations regarding the age range of the patient were made by examining OPGs. Exact age was not estimated.

Bosmans et $\mathrm{al}^{14}$ using OPGs in a study, selected 6 teeth and performed age estimation using criteria such as maximum tooth length, pulp length, and root and pulp width. They classified the study population, whose ages ranged from 29 to 70 years, into decades with time intervals of 9 years. The age estimations were reported to be convenient to the decades of chronological ages, based on a single dentist's evaluation.

The criteria used in our study were a compi- lation derived from the literature; however, the manner of evaluation in this study was different. The authors were inspired by previous studies related to the subject in order to take a further step. OPGs were evaluated considering the age groups of the study population, which is concordant with the previous studies performing evaluation according to decades. ${ }^{12}$ In parallel to the literature, author should suggest the use of skeletal measurements in addition to dental methods, for accurate age estimation. ${ }^{7}$

\section{CONCLUSIONS}

Despite the variations related to the practitioners, in this study, there were no significant differences in age estimation between both two participant practitioners. Age estimation through the evaluation of OPGs revealed the most reliable results for the first decade of life. However, the method showed unreliable results for the fourth decade.

The reliability of age estimation reduced in older age groups. The possible reasons might be the reduction of the criteria and signs for the age estimation of OPGs in older persons and the variability of the oral health status of patients in older age groups.

Age estimation with OPGs can be used to make a significant percentage of forecasts in areas such as forensic medicine and forensic dentistry, especially in young patients. In order to achieve accurate and reliable age estimation, in addition to millimetric measurements of the teeth, skeletal measurements and examinations should be performed.

\section{REFERENCES}

1. Schmeling A, Geserick G, Reisinger W, Olze A. Age estimation. Forensic Sci Int 2007;165:178-181.

2. Willems G. A review of the most commonly used dental age estimation techniques. J Forensic Odontostomatol 2001;19:9-17.

3. Hillson S, Dental Anthropology, Cambridge University Press, Cambridge, 1996.

4. Pelsmaekers B, Loos R, Carels C, Derom C, Vlietinck R. The genetic contribution to dental maturation. J Dent Res 1997; 76:1337-1340.

5. Mörnstad H, Staaf V, Welander U. Age estimation with aid of tooth development a new method based on objective measurements. Scand J Dent Res 1994;102:137-143. 
6. Gustafson G. Age determination on teeth. J Am Dent Assoc 1950;41:45-54.

7. Koshy S, Tandon S. Dental age assessment: the applicability of Demirjian's method in South Indian children. Forensic Sci Int 1998;94:73-85.

8. Prapanpoch S, Dove SB, Cottone JA. Morphometric analysis of the dental pulp chamber as a method of age determination in humans. Am J Forensic Med Pathol 1992;13:50-55.

9. Noble HW. The age estimation of age from the dentition. $J$ Forensic Sci Soc 1974;14:215-221.

10. Pindborg JJ. Pathology of the dental hard tissues. Copenhagen: Munksgaard, 1970;294-325.

11. Kvaal SI, Kolveitt KM, Thomsen IO, Solheim T. Age estimations of adults from dental radiographs. Forensic Science Int 1995;74:175-185.

12. Paewinsky E, Pfeiffer H, Brinkmann B. Quantification of secondary dentine formation from orthopantomograms--a contribution to forensic age estimation methods in adults. Int J Legal Med 2005;119:27-30.

13. Johansan G. Age determination from teeth. Odontol Revy 1971;22:1-126.

14. Bosmans N, Ann P, Aly M, Willems G. The application of Kvaal's dental age calculation technique on panoramic dental radiographs. Forensic Sci Int 2005;29:208-212.

15. Maber M, Liversidge HM, Hector MP. Accuracy of age estimation of radiographic methods using developing teeth. Forensic Sci Int 2006;159S:68-73.

16. Du Chesne A, Benthaus S, Teige K, Brinkmann B. Postmortem orthopantomography-an aid in screening for identification purposes. Int J Legal Med 2000;113:63-69.

17. Tunc ES, Koyuturk AE. Dental age assessment using Demirjian's method on northern Turkish children. Forensic Sci Int 2008; 175:23-26.

18. Kvaal SI, Kolltveit KM, Thomsen IO, Solheim T. Age estimation of adults from dental radiographs. Forensic Sci Int 1995;74:175-85.

19. Kim YK, Kho HS, Lee KH. Age estimation by occlusal tooth wear. J Forensic Sci 2000;45:303-309.

20. Solheim T. Amount of secondary dentin as an indicator of age. Scand J Dent Res 1992;100:193-199. 